\title{
Poorer sleep quality among adult patients with pectus excavatum in Taiwan: A pilot study
}

\author{
Yeung-Leung Cheng, MD, PhD, ${ }^{\text {a,b }}$ Chou-Chin Lan, MD, PhD, ${ }^{\text {b,c }}$ Yao-Kuang Wu, MD, ${ }^{\text {,c }}$ \\ Wen-Lin Su, MD, PhD, ${ }^{\mathrm{b}, \mathrm{c}}$ and Mei-Chen Yang, $\mathrm{MD}^{\mathrm{b}, \mathrm{c}}$
}

\section{ABSTRACT}

Objectives: Patients with pectus excavatum have a poorer quality of life than the general population. Sleep quality is a critical component of quality of life. This pilot study compared the sleep quality of adult patients with pectus excavatum with that of the general population in Taiwan.

Methods: Participants comprised 30 healthy adults, 21 adult outpatients with pectus excavatum, and 33 adults with pectus excavatum receiving Nuss surgery. The Pittsburgh Sleep Quality Index measured subjective sleep quality; the Visual Analog Scale for Pain, Brief Symptom Rating Scale-5, Beck Depression Inventory II, and 36-Item Short Form Survey measured quality of life; and the Epworth Sleepiness Scale measured excessive daytime sleepiness. Outcome measurements were obtained at baseline for all groups and 6 months after Nuss surgery for inpatients.

Results: Patients with pectus excavatum had poorer sleep quality and worse quality of life than controls. Poorer sleep quality was not related to the severity of pectus excavatum per se, but to psychologic issues (beta $=0.18, P<.001$ in multiple linear regression analysis). Measurement time had no effect on sleep quality (beta $=0.31, P=.594$ for outpatient group; beta $=0.27, P=.665$ for inpatients). Sleep quality of patients with pectus excavatum improved after Nuss surgery (Pittsburgh Sleep Quality Index: 4 vs 7, $P=.002$ ).

Conclusions: Our findings suggested an association between poor sleep quality and pectus excavatum; thus, sleep quality should be evaluated before and after the Nuss procedure. (J Thorac Cardiovasc Surg 2019;157:769-80)

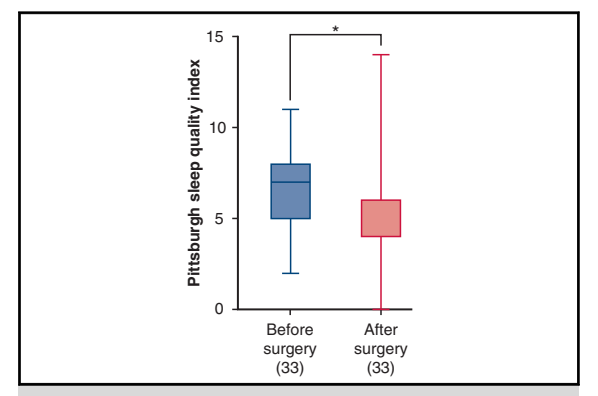

Surgical repair could improve poorer sleep quality for patients with $\mathrm{PE}$.

\section{Central Message}

Regardless of disease severity, PE negatively affects psychologic function, which, in turn, affects sleep quality. Surgical repair could improve sleep quality for patients with $\mathrm{PE}$.

\section{Perspective}

Many studies have shown that patients with $\mathrm{PE}$ have a poor QOL. On average, humans spend one fourth to one third of the day asleep. Therefore, sleep quality should be included while evaluating QOL. However, sleep quality has never been studied in patients with PE. Our findings suggested a potential association between poor sleep quality and PE.

See Editorial Commentaries pages 781 and 782.

\footnotetext{
From the ${ }^{\mathrm{a}}$ Division of Thoracic Surgery, Department of Surgery, and ${ }^{\mathrm{c}}$ Division of Pulmonary Medicine, Department of Internal Medicine, Taipei Tzu Chi Hospital, Buddhist Tzu Chi Medical Foundation, New Taipei City, Taiwan; and ${ }^{\text {b School of }}$ Medicine, Tzu Chi University, Hualien, Taiwan.

The study was supported by a grant from the Taipei Tzu Chi Hospital, Buddhist Tzu Chi Medical Foundation (TCRD-TPE-105-36).

The Institutional Review Board of Taipei Tzu Chi Hospital, Buddhist Tzu Chi Medical Foundation approved this report (Protocol No: 04-XD15-056 at August 20, 2015, and 05-XD57-110 at February 20, 2017). Informed consent was obtained from all study participants.

Received for publication Dec 15, 2017; revisions received June 29, 2018; accepted for publication July 14, 2018; available ahead of print Sept 6, 2018.

Address for reprints: Mei-Chen Yang, MD, Division of Pulmonary Medicine, Department of Internal Medicine, Taipei Tzu Chi Hospital, Buddhist Tzu Chi Medical Foundation, New Taipei City, Taiwan No 289, Jianguo Rd, Xindian Dist, New Taipei City 23143, Taiwan (E-mail: mimimai3461@gmail.com).

0022-5223

Copyright (C) 2018 by The American Association for Thoracic Surgery. Published by Elsevier Inc. This is an open access article under the CC BY-NC-ND license (http:// creativecommons.org/licenses/by-nc-nd/4.0/).

https://doi.org/10.1016/j.jtcvs.2018.07.050
}

Patients with pectus excavatum (PE) have a lower quality of life (QOL) than the general population. ${ }^{1-3}$ Most QOL studies on PE primarily focused on children and adolescents, but not adults. Only a few studies that included both adolescent and adult patients with $\mathrm{PE}$ compared their QOL with that of healthy subjects. ${ }^{4-6}$ To

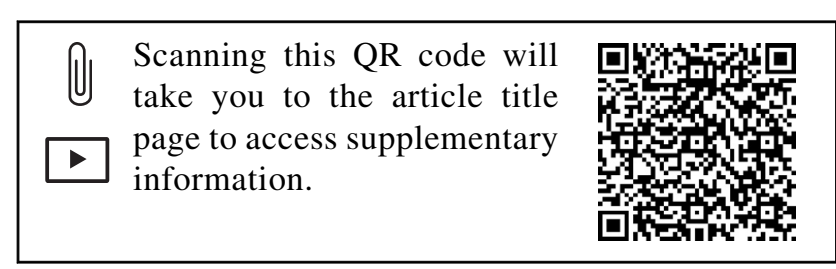




$\begin{array}{ll}\text { Abbreviations and Acronyms } \\ \text { BDI-II } & =\text { Beck Depression Inventory version II } \\ \text { BP } & =\text { bodily pain } \\ \text { BSRS-5 } & =\text { Brief Symptom Rating Scale-5 } \\ \text { GH } & =\text { general health } \\ \text { MCS } & =\text { mental component score } \\ \text { MH } & =\text { mental health } \\ \text { NBS }(\%) & =\text { norm-based score }(0-100) \\ \text { PCS } & =\text { physical component score } \\ \text { PE } & =\text { pectus excavatum } \\ \text { PF } & =\text { physical functioning } \\ \text { PSG } & =\text { polysomnography } \\ \text { PSQI } & =\text { Pittsburgh Sleep Quality Index } \\ \text { QOL } & =\text { quality of life } \\ \text { RE } & =\text { role emotional } \\ \text { RP } & =\text { role physical } \\ \text { SF } & =\text { social functioning } \\ \text { SF-36v2 } & =36-\text { Item Short Form Survey Version } 2 \\ \text { VAS } & =\text { visual analog scale for pain } \\ \text { VT } & =\text { vitality }\end{array}$

date, no study has globally evaluated QOL in adult patients with PE aged more than 20 years.

On average, humans spend one fourth to one third of the day asleep. Betty Ferrell and Marcia Grant developed a model to evaluate QOL that includes sleep quality for patients with various diseases. ${ }^{7,8}$ Two previous studies have used this model to investigate the impact of Nuss surgery on QOL (including sleep quality) of children with PE and found improved postsurgery QOL and sleep quality. ${ }^{9,10}$ However, this model subjectively evaluated sleep quality using only a 10-point rating scale that may be too arbitrary.

The 36-Item Short Form Survey (SF-36) is used worldwide to evaluate health-related QOL. However, only 1 study used the SF-36 to evaluate health-related QOL in patients with PE. ${ }^{11}$

The Pittsburgh Sleep Quality Index (PSQI) is well known for evaluating subjective sleep quality. ${ }^{12}$ The PSQI is negatively correlated with SF-36. ${ }^{13,14}$ However, to date, no studies have used the PSQI to evaluate sleep quality for patients with PE.

The Beck Depression Inventory-II (BDI-II) is a useful tool to subjectively evaluate psychologic outcomes of illness. ${ }^{15}$ The Brief Symptom Rating Scales-5 (BSRS-5) can reliably screen for psychiatric morbidities in both medical and community settings. ${ }^{16}$ Most studies on PE used modified Nuss questionnaires or other disease-specific questionnaires to evaluate the psychologic impact of PE. ${ }^{1,2,4-6,11,17-19}$ However, no studies on PE have used the BDI-II and BSRS-5.
This study attempted to evaluate (1) sleep quality and QOL in adult patients with PE and the general population; (2) factors affecting sleep quality in adult patients with PE; and (3) if Nuss surgery could improve sleep quality for adult patients with PE.

\section{MATERIALS AND METHODS Participants}

A total of 33 adult patients with PE scheduled for the Nuss procedure were recruited at the Division of Thoracic Surgery, Department of Surgery, Taipei Tzu-Chi Hospital, in January to December 2016 as the inpatient group. Inclusion criteria were age 20 years or more with a Haller index 3.0 or more, no psychiatric or medical illness, no use of psychoactive, soporific, or illegal drugs, and nonsmoking. The control group comprised 30 healthy adult nonsmokers recruited from the families of patients with PE, medical staff, and the families of medical staff who fulfilled the same inclusion criteria but had no PE confirmed by chest surgeon. To control for the effect of presurgery stress in the inpatient group during the baseline outcome measurement, we extracted 21 age-matched adults with PE from the outpatient department when they were first evaluated. These patients were enrolled in another study of ours (Institutional Review Board protocol No.: 05-X17-070 with ClinicalTrials.gov ID.: NCT03346876), were not under consideration for Nuss surgery, and served as the outpatient group. All participants completed questionnaires at baseline, but only the inpatient group had second measurements 6 months after Nuss surgery (Video 1).

All participation was voluntary, and written informed consent was obtained. The study was approved by the institutional review board of Taipei Tzu Chi Hospital, Buddhist Tzu Chi Medical Foundation (Institutional Review Board protocol No.: 04-XD15-056).

\section{Anthropometric Measurements and Demographic Data}

Baseline characteristics were collected, including age, gender, body height, body weight, body mass index $\left(\mathrm{kg} / \mathrm{m}^{2}\right)$, indications of surgery in inpatient group and presetting symptoms in outpatient group, Haller index, ${ }^{20}$ and employment status (student or nonstudent).

\section{Measurements of Quality of Life}

QOL was assessed using a visual analog scale for pain (VAS), the Chinese version of the BSRS- $5^{16}$, and validated Chinese versions of the BDI$\mathrm{II}^{21}$ and SF-36v2 (Optum SF-36v2 Health Survey) (Table E1).

The VAS is a simple tool to subjectively rate the severity of painful bodily sensations, ranging from 0 to $10(0=$ no pain; $10=$ extremely severe pain).

The BSRS- $5^{16}$ is a 5 -item, self-administered questionnaire measuring (1) anxiety (feeling tense or high-strung), (2) depression (feeling depressed or in a low mood), (3) hostility (feeling easily annoyed or irritated), (4) interpersonal sensitivity (feeling inferior to other people), and (5) additional symptoms (having trouble falling asleep in the past week). Each item of the BSRS-5 is rated on a scale from 0 to $4(0=$ not at all; $1=$ a little bit; $2=$ moderately; 3 = quite a bit; $4=$ extremely). The total score of BSRS-5 represents the psychologic status during the day of measurement.

The 21-item Chinese BDI-II measures the severity of self-reported depression. Each item is scored from 0 to $3(0=\mathrm{I}$ do not feel so; $1=\mathrm{I}$ feel so; 2 = I feel so all the time and I can't snap out of it; $3=$ I feel so, that I can't stand it). The sum of the scores of the 21 items (BDI-II score) indicates the severity of depressive symptoms (higher BDI-II score indicating more severe depressive symptoms).

The SF-36v2 contains 8 health domains (general health [GH], physical functioning [PF], role physical [RP], bodily pain [BP], vitality [VT], social functioning $[\mathrm{SF}]$, role emotional $[\mathrm{RE}]$, and mental health $[\mathrm{MH}]$ ) and provides a physical component score (PCS), including GH, PF, RP, BP, and 


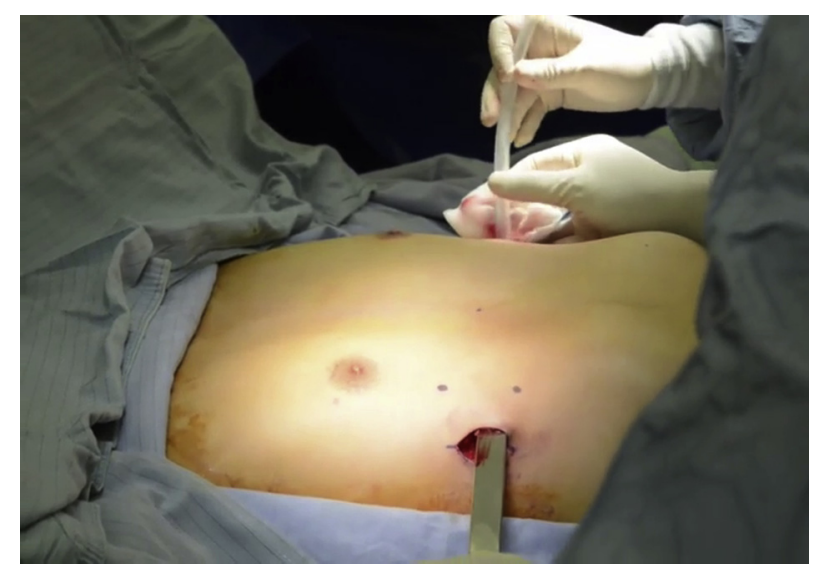

VIDEO 1. This is a case of a 29 -year-old man receiving a modified Nuss procedure using bilateral thoracoscopic assistance. Lots of studies had showed that patients with PE had a poor QOL, which can be improved after surgical correction. On average, humans spend one fourth to one third of the day asleep. We suggested sleep quality should be included while evaluating QOL. However, sleep quality had never been studied in patients with PE. Therefore, we conducted a pilot clinical study to evaluate if sleep quality is affected by PE and if surgical correction could improve patients' sleep quality. Our findings suggested sleep quality is poorer in patients with PE in Taiwan that can be attributed to the resulting psychologic issues, rather than the PE itself. The poorer sleep quality could be improved after Nuss repair surgery for correction of $\mathrm{PE}$. At present, sleep quality is not routinely evaluated in patients with $\mathrm{PE}$ before and after surgical correction. We suggested both subjective or objective evaluations of sleep quality might be considered before and after a patient undergoes surgical correction for PE. Video available at: https://www.jtcvs.org/ article/S0022-5223(18)32041-5/fulltext. a mental component summary score (MCS), including VT, SF, RE, and $\mathrm{MH}$. All component scores were presented as percentages using $t$ scoring, which is norm-based scoring (NBS) that allows comparisons with other generic health surveys. Each scale is scored to have a mean of 50 and a standard deviation of 10 reflecting the general US population in 2009. Higher scores indicate better functioning and well-being.

\section{Measurements of Sleep Quality}

The validated Chinese version of PSQI was used to assess subjective sleep quality during the past 1 month (Table E1).22 The scale comprises 19 items with 7 components: sleep quality (PSQ-1), sleep latency (PSQ2), sleep duration (PSQ-3), sleep efficiency (PSQ-4), sleep disturbance (PSQ-5), frequency of sleeping medication use (PSQ-6), and daytime functional impairment (PSQ-7). Each PSQ item is rated on a scale from 0 to 3 $(0=$ no difficulty, $3=$ extreme difficulty $)$. The global sleep quality index was calculated with PSQI total scores, ranging from 0 to 21. Good sleep quality was defined as PSQI total scores 5 or less, and poor sleep quality was defined as PSQI total scores 6 or greater.

\section{Statistical Analysis}

Raw data of the SF-36v2 Chinese version were analyzed using the Health Outcomes Scoring Software 5.0 provided by Optum. Continuous data were summarized as mean \pm standard deviation or median (interquartile rang, $\mathrm{P}_{25}-\mathrm{P}_{75}$ ); categoric data were summarized as count (\%). Differences between groups were compared using analysis of variance, Wilcoxon rank-sum test, and Kruskal-Wallis test for parametric/nonparametric continuous data, and Pearson chi-square test for categoric data. If a statistically significant difference was found after analysis of variance, post hoc tests were conducted using Tukey's test. Dunn's test was implemented if a significant difference was obtained for the Kruskal-Wallis test. Differences between pre-test and post-test were compared using paired $t$ test and Wilcoxon signed-rank test for continuous data, and McNemar's test for categoric data. Spearman correlation analysis was performed for the

TABLE 1. Characteristics of the control, outpatient, and inpatient groups

\begin{tabular}{|c|c|c|c|c|}
\hline Variables & $\begin{array}{c}\text { Control } \\
(n=30)\end{array}$ & $\begin{array}{c}\text { Outpatient } \\
(\mathbf{n}=\mathbf{2 1})\end{array}$ & $\begin{array}{c}\text { Inpatient } \\
(n=33)\end{array}$ & $P$ value \\
\hline Surgery indication/presenting symptoms & & & & $<.001^{*}$ \\
\hline Noncosmetic, n (\%) & - & $10(47.6 \%)$ & $30(90.9 \%)$ & \\
\hline Cosmetic, n (\%) & - & $11(52.4 \%)$ & $3(9.1 \%)$ & \\
\hline Haller index & - & $3.5(3.3,4.0)$ & $3.6(3.3,4.2)$ & .392 \\
\hline Gender & & & & - \\
\hline Male, n (\%) & $21(70.0 \%)$ & $21(100.0 \%)$ & $28(84.8 \%)$ & \\
\hline Female, n (\%) & $9(30.0 \%)$ & $0(0.0 \%)$ & $5(15.2 \%)$ & \\
\hline Age, y & $25.5(22.0,30.0)$ & $24.0(23.0,30.0)$ & $24.0(23.0,30.0)$ & .916 \\
\hline Body height, $\mathrm{cm}$ & $171.1 \pm 8.7$ & $176.0 \pm 6.4$ & $173.6 \pm 7.4$ & .086 \\
\hline Body weight, $\mathrm{kg}$ & $63.2 \pm 10.4$ & $61.8 \pm 6.0$ & $60.8 \pm 8.9$ & .572 \\
\hline Body mass index, $\mathrm{kg} / \mathrm{m}^{2}$ & $21.6 \pm 2.6$ & $19.9 \pm 1.5$ & $20.3 \pm 2.7$ & .035 \\
\hline Occupation & & & & .700 \\
\hline Non-student, n (\%) & $18(60.0 \%)$ & $13(61.9 \%)$ & $23(69.7 \%)$ & \\
\hline Student, n $(\%)$ & $12(40.0 \%)$ & $8(38.1 \%)$ & $10(30.3 \%)$ & \\
\hline
\end{tabular}

Data on Haller index and age are presented as median (interquartile range); body height, body weight, and body mass index are shown as mean \pm standard deviation. Surgery indication, gender, and occupation are expressed as count (\%). Dash indicates no available data. Continuous data were analyzed using analysis of variance, Wilcoxon rank-sum test, and Kruskal-Wallis test; categoric data were analyzed using Pearson chi-square test; post hoc tests were conducted using Tukey's test and Dunn's test. *Compared with outpatient and inpatient group. Significantly different between outpatient and inpatient groups, $P<.01$. 
TABLE 2. Comparison of quality of life, sleep quality, and daytime sleepiness between control, outpatient, and inpatient groups

\begin{tabular}{|c|c|c|c|c|}
\hline Variables & $\begin{array}{c}\text { Control } \\
(\mathbf{n}=\mathbf{3 0})\end{array}$ & $\begin{array}{c}\text { Outpatient } \\
(\mathbf{n}=\mathbf{2 1})\end{array}$ & $\begin{array}{c}\text { Inpatient } \\
(\mathrm{n}=\mathbf{3 3})\end{array}$ & $P$ value \\
\hline \multicolumn{5}{|l|}{ QOL } \\
\hline VAS scores & $0(0,0) \dagger$ & $0(0,2)^{*}$ & $0(0,2)$ & .003 \\
\hline BSRS-5 & $2.5(0.0,4.0)$ & $2.0(2.0,5.0)$ & $3.0(1.0,6.0)$ & .190 \\
\hline BDI-II & $1(0,2) \ddagger$ & $3(1,6)$ & $7(3,11)^{*}$ & $<.001$ \\
\hline \multicolumn{5}{|l|}{ SF-36v2 } \\
\hline PCS, $\%$ & $57.8(54.3,59.9) \ddagger$ & $54.1(47.5,57.2)$ & $52.7(49.3,55)^{*}$ & .001 \\
\hline MCS, $\%$ & $51.9 \pm 5.2 \ddagger$ & $48.4 \pm 7.1$ & $43.5 \pm 8.5^{*}$ & $<.001$ \\
\hline PF_NBS, \% & $57.5(55.6,57.5) \dagger, \dagger$ & $53.7(49.9,55.6)^{*}$ & $53.7(49.9,55.6)^{*}$ & $<.001$ \\
\hline RP_NBS, $\%$ & $54.9(50.4,57.2) \ddagger$ & $52.7(43.7,57.2)$ & $43.7(39.2,50.4)^{*}$ & $<.001$ \\
\hline BP_NBS, \% & $62.0(51.5,62.0)$ & $55.6(55.6,62.0)$ & $55.6(51.5,62.0)$ & .712 \\
\hline GH_NBS, $\%$ & $57.9(53.2,62.7) \dagger, \dagger$ & $48.4(41.3,49.9)^{*}$ & $47.5(38.0,52.2)^{*}$ & $<.001$ \\
\hline VT_NBS, \% & $53.7 \pm 7.1$ & $50.5 \pm 7.5$ & $48.5 \pm 8.8$ & .040 \\
\hline SF_NBS, $\%$ & $57.3(52.3,57.3) \ddagger$ & $52.3(47.3,57.3)$ & $47.3(47.3,52.3)^{*}$ & $<.001$ \\
\hline RE_NBS, \% & $56.2(56.2,56.2) \ddagger$ & $56.2(42.2,56.2)$ & $42.2(38.8,49.2)^{*}$ & $<.001$ \\
\hline MH_NBS, \% & $50.9 \pm 5.8 \ddagger$ & $47.8 \pm 5.8$ & $44 \pm 8.5^{*}$ & $<.001$ \\
\hline \multicolumn{5}{|l|}{ Sleep quality } \\
\hline PSQI-total scores & $4.4 \pm 2.4 \ddagger$ & $5.4 \pm 2.2$ & $6.6 \pm 2.3^{*}$ & .002 \\
\hline PSQI-severity & & & & .028 \\
\hline Good sleep quality, n (\%) & $21(70.0 \%)$ & $11(52.4 \%)$ & $12(36.4 \%)$ & \\
\hline Poor sleep quality, n (\%) & $9(30.0 \%)$ & $10(47.6 \%)$ & $21(63.6 \%)$ & \\
\hline PSQ-1 & $1(1,1)$ & $1(1,2)$ & $1(1,2)$ & .081 \\
\hline PSQ-2 & $1(0,1) \dagger$ & $1(1,2)$ & $2(1,2)^{*}$ & .001 \\
\hline PSQ-3 & $1(0,2)$ & $0(0,1)$ & $1(0,2)$ & .320 \\
\hline PSQ-4 & $0(0,0)$ & $0(0,0)$ & $0(0,0)$ & - \\
\hline PSQ-5 & $1(1,1)$ & $1(1,1)$ & $1(1,2)$ & .012 \\
\hline PSQ-6 & $0(0,0)$ & $0(0,0)$ & $0(0,0)$ & - \\
\hline PSQ-7 & $1(0,1)$ & $1(0,1)$ & $1(1,2)$ & .175 \\
\hline \multicolumn{5}{|l|}{ Daytime sleepiness } \\
\hline ESS score, points & $5.6 \pm 3.1$ & $6.9 \pm 4.0$ & $8.4 \pm 4.5$ & .018 \\
\hline Excessive daytime sleepiness & & & & .053 \\
\hline No, ESS score $\leq 10, \mathrm{n}(\%)$ & $29(96.7)$ & $18(85.7)$ & $25(75.8)$ & \\
\hline Yes, ESS score $>10, \mathrm{n}(\%)$ & $1(3.3)$ & $3(14.3)$ & $8(24.2)$ & \\
\hline
\end{tabular}

Data on MCS, VT_NBS, MH_NBS, PSQI-total scores, and ESS score are presented as mean \pm standard deviation; other continuous variables are shown as median (interquartile range). PSQI-severity and excessive daytime sleepiness are expressed as count (\%). Continuous data were analyzed using analysis of variance and Kruskal-Wallis test; categoric data were analyzed using Pearson chi-square test; post hoc tests were conducted using Tukey's test and Dunn's test. QOL, Quality of life; VAS, visual analog scale; BSRS-5, Brief Symptom Rating Scale; BDI-II, Beck Depression Inventory; SF-36v2, 36-Item Short Form Survey Chinese Version 2; PCS, physical component score; MCS, mental component score; $P F$, physical functioning; $\_N B S$, norm-based score (0-100\%); $R P$, role physical; $B P$, bodily pain; $G H$, general health; $V T$, vitality; $S F$, social functioning; $R E$, role emotional; $M H$, mental health; PSQI total score, sum of PSQ-1 to PSQ-7; PSQI severity, good sleep quality for PSQI total score $\leq 5$ and poor sleep quality for PSQI total score $\geq 6$; $P S Q-1$, Pittsburgh sleep quality questionnaire-1 (subjective sleep quality) (0-3); $P S Q-2$, Pittsburgh sleep quality questionnaire-2 (subjective sleep latency) (0-3); $P S Q-3$, Pittsburgh sleep quality questionnaire-3 (subjective sleep duration) (0-3); $P S Q-4$, Pittsburgh sleep quality questionnaire-4 (subjective sleep efficiency) (0-3); $P S Q-5$, Pittsburgh sleep quality questionnaire-5 (subjective sleep disturbance) (0-3); PSQ-6, Pittsburgh sleep quality questionnaire-6 (sleep pills usage frequency) (0-3); $P S Q-7$, Pittsburgh sleep quality questionnaire-7 (daytime functional impairment) (0-3); ESS, Epworth sleepiness scale. *Significantly different between control group and a given group, $P<.01$. $\dagger$ Significantly different between outpatient group and a given group, $P<.01$. $\ddagger$ Significantly different between inpatient group and a given group, $P<.01$. Bold indicates there was difference between 2 given groups.

correlation of the Haller index with subjects' characteristics, and the measure of the correlation between the 2 variables was presented as a Spearman correlation coefficient (rho). A high correlation was defined as 0.7 to 1.0 , (or -0.7 to -1.0 ), medium correlation as 0.3 to 0.7 (or -0.3 to -0.7 ), and low correlation as 0.1 to 0.3 (or -0.1 to -0.3 ). A linear regression model was built to analyze factors of the PSQI total score. Variables having a $P$ value less than .01 in the univariate analysis were selected and evaluated by multivariate analysis with stepwise selection. All statistical assessments were 2-tailed. Statistical analyses were carried out with R Core Team (2016; A language and environment for statistical computing, R Foundation for Statistical Computing, Vienna, Austria, https://www.R-project. org/) and SAS version 9.4 (SAS Institute Inc, Cary, NC).

\section{RESULTS}

Table 1 provides a description of the characteristics of the 3 groups. There was no significant difference in age between groups (mean age $=24$ years for inpatients and outpatients; mean age $=25.5$ years for controls). Most were male $(100.0 \%$ in outpatient group and $84.8 \%$ in inpatient group) and nonstudents $(61.9 \%$ in the outpatient group and $69.7 \%$ in the inpatient group, $P>.01$ ). Compared with the outpatient group, the inpatient group was significantly higher in noncosmetic symptoms/surgical 

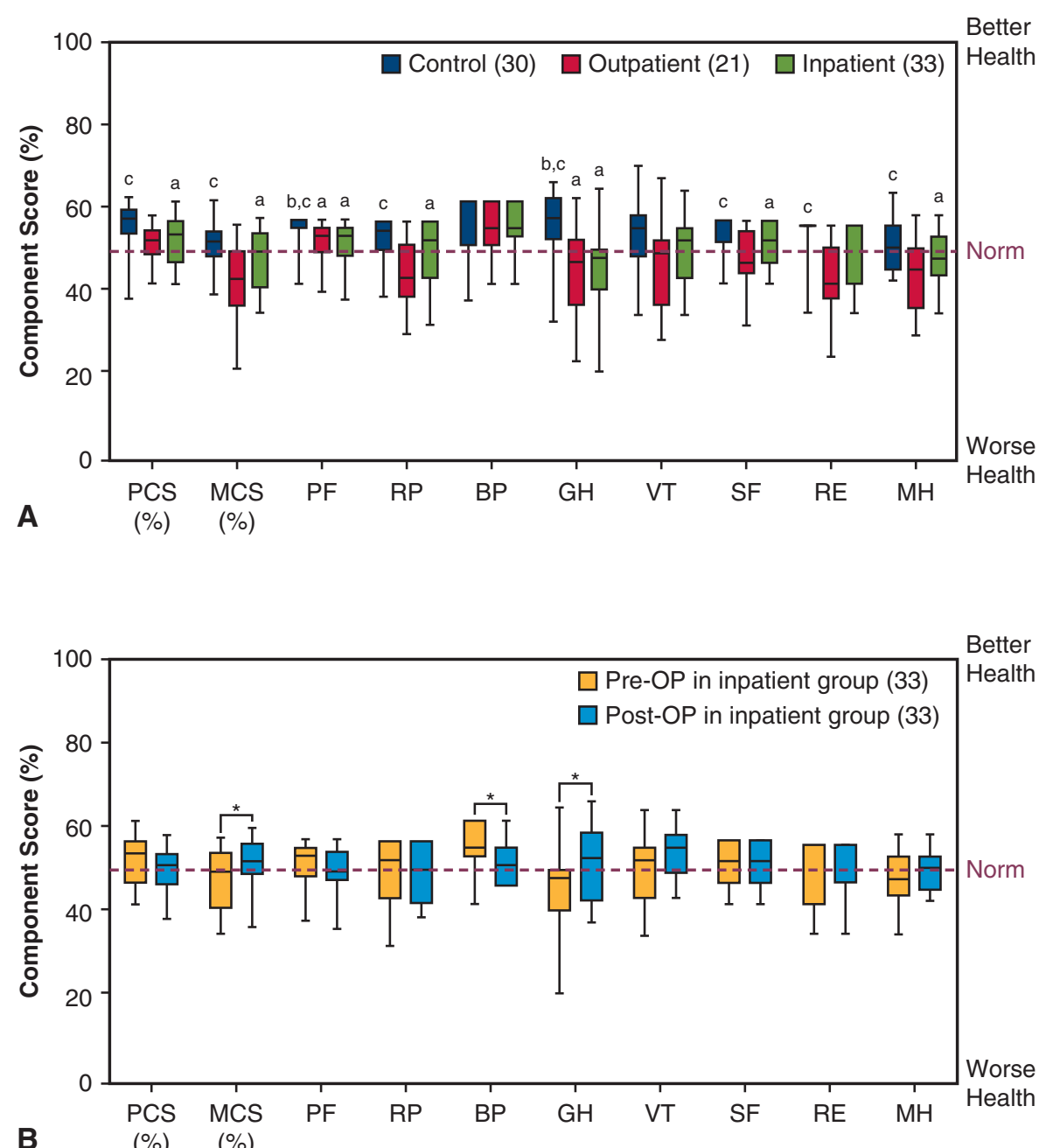

FIGURE 1. A, Comparison of QOL (SF-36 V2) among the control, outpatient, and inpatient groups at baseline. The inpatient group had worse PCS (including worse PF, RP, GH components) and MCS (including SF, RE, and MH) than the control group. The outpatient group had worse PF and GH than the control group (minimum, maximum, median, Q1 and Q3 values are presented in box-and-whisker plots). a, Significantly different between control group and a given group, $P<.01 . \mathrm{b}$, Significantly different between outpatient group and a given group, $P<.01$. c, Significantly different between inpatient group and a given group, $P<.01 .{ }^{*} P<.01$. $P C S(\%)$, Physical component score (0-100); MCS $(\%)$, mental component score (0-100); $P F$, physical functioning norm-based score (0-100); $R P$, role physical norm-based score (0-100); $B P$, bodily pain norm-based score (0-100); $G H$, general health norm-based score (0-100); $V T$, vitality norm-based score (0-100); $S F$, social functioning norm-based score (0-100); $R E$, role emotional norm-based score (0-100); $M H$, mental health norm-based score (0-100). B, Comparison of QOL (SF-36 V2) before and after Nuss surgery in the inpatient group. Six months after Nuss surgery, MCS and GH improved, but BP was worse (minimum, maximum, median, Q1 and Q3 values are presented in box-and-whisker plots.) $* P<.01$, significant difference between 2 groups.

indications $(47.6 \%$ vs $90.9 \%, P<.01)$. Other characteristics showed no differences among the 3 groups.

Table 2 shows the comparison of QOL and sleep quality among the 3 groups. All scales (except for BSRS-5, BP and VT) showed significant differences among the 3 groups (all $P<.01)$. In the inpatient group, the PCS, MCS, PF, RP, GH, $\mathrm{SF}, \mathrm{RE}$, and $\mathrm{MH}$ were significantly worse than in the control group (all $P<.01$, Table 2 and Figure $1, A$ ). In both the outpatient and inpatient groups, $\mathrm{PF}$ and $\mathrm{GH}$ were significantly worse than in the control group (all $P<.01$, Table 2 and Figure 1, A). The VAS pain scale was significantly lower in the control group than the outpatient groups (all $P<.01$, Table 2).

The PSQI total scores of the inpatient group were higher than that of the control group $(6.6 \pm 2.3$ vs $4.4 \pm 2.4$, $P<.01$, Table 2 and Figure 2, left ), suggesting the inpatient group had poorer subjective sleep quality than the control group in the past month. In addition, the subjective sleep latency (PSQ-2 scores) of the inpatient groups was longer (indicating patients took more time falling asleep) than for the control group $(P \leq .01$, Table 2$)$, suggesting that the inpatient group spent more time falling asleep in the 

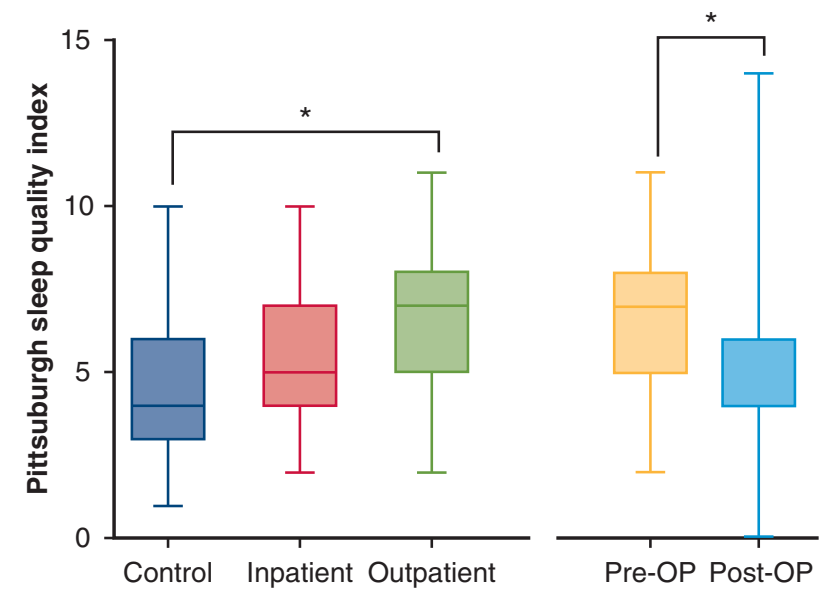

(30)

(33)

(33)
(33)

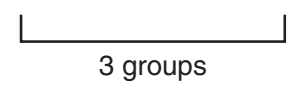

$\begin{array}{lrrrrr}\text { Min } & 1 & 2 & 2 & 2 & 0 \\ \text { Q1 } & 3 & 4 & 5 & 5 & 4 \\ \text { Median } & 4 & 5 & 7 & 7 & 4 \\ \text { Q3 } & 6 & 7 & 8 & 8 & 6 \\ \text { Max } & 10 & 10 & 11 & 11 & 14\end{array}$

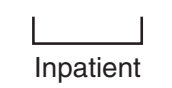

FIGURE 2. Comparison of PSQI total scores among control, outpatient, and inpatient groups at baseline (left) before and 6 months after Nuss surgery only in the inpatient group (right). The sleep quality of the inpatient group was poorer than that of the control group (left) and could be improved by Nuss surgery (right). Minimum, maximum, median, Q1, and Q3 values are presented in box-and-whisker plots and the measurements results were also shown under the figure. ${ }^{*} P<.01$, significant difference between 2 groups.

past. For excessive daytime sleepiness, there were no significantly differences among the 3 groups.

Sleep quality and QOL were compared before and after Nuss surgery in the inpatient group, as shown in Table 3. After Nuss surgery, the pain significantly increased (VAS scores increased from 0 vs $2, P<.001$, and SF-36v2 BP worse from $55.6 \%$ vs $51.5 \%, P=.001$ ). However, BDIII scores significantly decreased (7 vs $4, P=.003$ ), and SF-36v2 MCS and GH significantly increased $(43.5 \% \pm 8.5 \%$ vs $48.0 \% \pm 8.3 \%, P=.001$ for MCS; $45.0 \% \pm 9.4 \%$ vs $50.6 \% \pm 8.2 \%, P=.002$ for $\mathrm{GH}$ ) (Table 3 and Figure 1, B). Total subjective sleep quality (PSQI total scores) significantly improved after Nuss surgery ( 7 vs $4, P=.002$; Table 3 and Figure 2, right). The PSQ-3 scores significantly decreased after Nuss surgery ( 1 vs $0, P=.001$, Table 3 ), suggesting that sleep duration significantly increased after Nuss surgery. The inpatient group also could sleep for longer periods after Nuss surgery.

Figure 3 shows correlations among characteristics, QOL, and sleep quality of the 3 groups. In the control group, the BDI-II had a medium correlation with SF36v2 PCS

TABLE 3. Comparison of quality of life, sleep quality, and daytime sleepiness before and after operation in inpatient group $(n=33)$

\begin{tabular}{|c|c|c|c|}
\hline Variables & Preoperation & Postoperation & $\begin{array}{c}P \\
\text { value }\end{array}$ \\
\hline \multicolumn{4}{|l|}{ QOL } \\
\hline VAS scores & $0(0,2)$ & $2(1,4)$ & $<.001$ \\
\hline BSRS-5 & $3(1,6)$ & $3(1,6)$ & .342 \\
\hline BDI-II & $7(3,11)$ & $4(1,7)$ & .003 \\
\hline \multicolumn{4}{|l|}{ SF-36v2 } \\
\hline PCS, \% & $52.1 \pm 3.8$ & $50.1 \pm 5.0$ & .070 \\
\hline MCS, $\%$ & $43.5 \pm 8.5$ & $48.0 \pm 8.3$ & .001 \\
\hline PF_NBS, $\%$ & $53.7(49.9,55.6)$ & $51.8(48.0,53.7)$ & .020 \\
\hline RP_NBS, \% & $43.7(39.2,50.4)$ & $43.7(41.4,48.2)$ & .911 \\
\hline BP_NBS, $\%$ & $55.6(51.5,62.0)$ & $51.5(46.7,55.6)$ & .001 \\
\hline GH_NBS, $\%$ & $45.0 \pm 9.4$ & $50.6 \pm 8.2$ & .002 \\
\hline VT_NBS, \% & $49.6(40.7,52.6)$ & $52.6(46.7,55.6)$ & .026 \\
\hline SF_NBS, \% & $47.3(47.3,52.3)$ & $52.3(47.3,57.3)$ & .062 \\
\hline RE_NBS, \% & $42.2(38.8,49.2)$ & $45.7(42.2,56.2)$ & .094 \\
\hline MH_NBS, $\%$ & $45.6(37.8,50.9)$ & $48.3(43.0,50.9)$ & .036 \\
\hline \multicolumn{4}{|l|}{ Sleep quality } \\
\hline PSQI-total scores & $7(5,8)$ & $4(4,6)$ & .002 \\
\hline PSQI-severity & & & .012 \\
\hline $\begin{array}{l}\text { Good sleep } \\
\text { quality, n (\%) }\end{array}$ & $12(36.4 \%)$ & $23(69.7 \%)$ & \\
\hline $\begin{array}{l}\text { Poor sleep } \\
\quad \text { quality, } \mathrm{n}(\%)\end{array}$ & $21(63.6 \%)$ & $10(30.3 \%)$ & \\
\hline PSQ-1 & $1(1,2)$ & $1(1,1)$ & .111 \\
\hline PSQ-2 & $2(1,2)$ & $1(1,2)$ & .231 \\
\hline PSQ-3 & $1(0,2)$ & $0(0,0)$ & .001 \\
\hline PSQ-4 & $0(0,0)$ & $0(0,0)$ & .863 \\
\hline PSQ-5 & $1(1,2)$ & $1(1,1)$ & .145 \\
\hline PSQ-6 & $0(0,0)$ & $0(0,0)$ & .500 \\
\hline PSQ-7 & $1(1,2)$ & $1(0,1)$ & .123 \\
\hline \multicolumn{4}{|l|}{ Daytime sleepiness } \\
\hline ESS score, points & $8.0(5.0,10.0)$ & $5.5(4.0,9.0)$ & .052 \\
\hline $\begin{array}{c}\text { Excessive daytime } \\
\text { sleepiness }\end{array}$ & & & .079 \\
\hline $\begin{array}{l}\text { No, ESS score } \\
\quad \leq 10, \mathrm{n}(\%)\end{array}$ & $25(75.8 \%)$ & $27(81.8 \%)$ & \\
\hline $\begin{array}{l}\text { Yes, ESS score } \\
>10, \mathrm{n}(\%)\end{array}$ & $8(24.2 \%)$ & $6(18.2 \%)$ & \\
\hline
\end{tabular}

Data on PCS, MCS, and GH_NBS are presented as mean \pm standard deviation; other continuous variables are shown as median (interquartile range). PSQI severity and excessive daytime sleepiness are expressed as count (\%). Continuous data were analyzed using paired $t$ test and Wilcoxon signed-rank test; categorical data were analyzed using McNemar's test. Bold indicates significant difference between preoperative and postoperative groups, $P<.01$. $Q O L$, Quality of life; VAS, visual analog scale; BSRS-5, Brief Symptom Rating Scale; BDI-II, Beck Depression Inventory; $S F$-36v2, 36-Item Short Form Survey Chinese Version 2; PCS, physical component score; $M C S$, mental component score; $P F$, physical functioning; $N N B S$, norm-based score (0-100\%); $R P$, role physical; $B P$, bodily pain; $G H$, general health; $V T$, vitality; $S F$, social functioning; $R E$, role emotional; $M H$, mental health; $P S Q I$ total score, sum of PSQ-1 to PSQ-7; PSQI severity, good sleep quality for PSQI total score $\leq 5$ and poor sleep quality for PSQI total score $\geq 6 ; P S Q-1$, Pittsburgh sleep quality questionnaire-1 (subjective sleep quality) (0-3); $P S Q-2$, Pittsburgh sleep quality questionnaire-2 (subjective sleep latency) (0-3); $P S Q-3$, Pittsburgh sleep quality questionnaire-3 (subjective sleep duration) (0-3); $P S Q-4$, Pittsburgh sleep quality questionnaire-4 (subjective sleep efficiency) (0-3); $P S Q-5$, Pittsburgh sleep quality questionnaire-5 (subjective sleep disturbance) (0-3); $P S Q-6$, Pittsburgh sleep quality questionnaire-6 (sleep pills usage frequency) (0-3); $P S Q-7$, Pittsburgh sleep quality questionnaire-7 (daytime functional impairment) (0-3); ESS, Epworth sleepiness scale. 
0.00 .51 .01 .52 .0
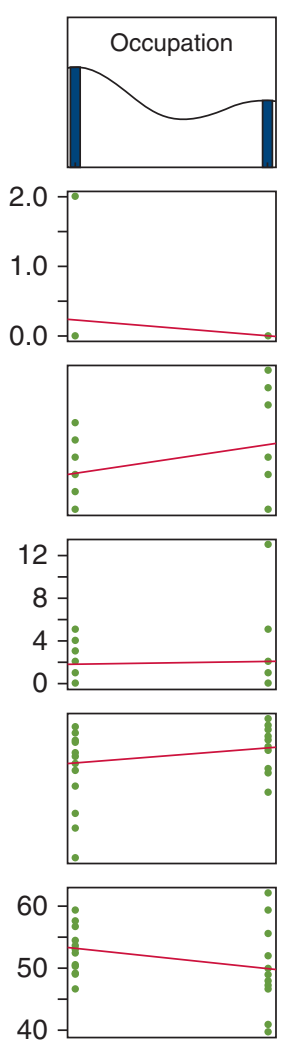

$40-$

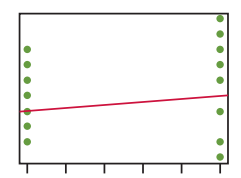

$\begin{array}{lll}0.0 & 0.4 & 0.8\end{array}$
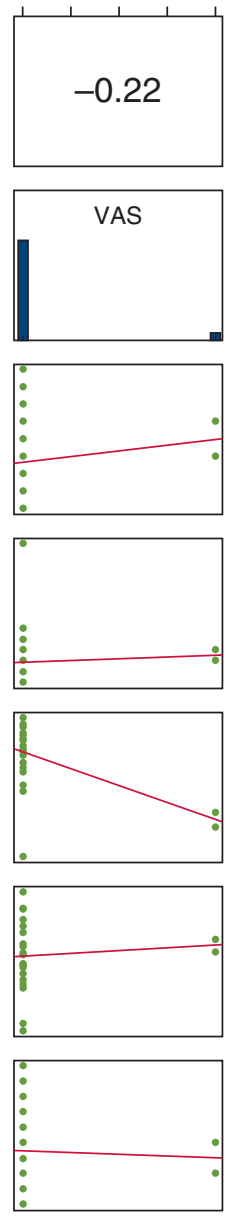

A

FIGURE 3. Spearman's correlation among participants' characteristics, QOL, and sleep quality of sleep in the 3 groups (A, control group; B, outpatient group; C, inpatient group). In the control group, the BDI-II had a medium correlation with PCS (A). In the outpatient group, the BSRS-5 had a medium correlation with the BDI-II and a high correlation with the MCS. The BDI-II had a medium correlation with the MCS. There was no correlation with the PSQI (B). In the inpatient group, the BSRS-5 had a medium correlation with the BDI-II and MCS. The BDI-II was highly correlated with the MCS. The PSQI had a medium correlation with both the MCS and BDI-II (C). $* P<.01$, significant correlation between 2 variables. VAS, Visual analog scale for pain; BSRS-5, Brief Symptom Rating Scale; BDI-II, Beck Depression Inventory; PCS (\%), physical component score (0-100); MCS (\%), mental component score (0-100); PSQI, Pittsburgh sleep quality index total scores.

(rho $=-0.48, P<.01)$. However, there was no correlation between total sleep quality (PSQI total scores) and all other variables in the control group (Figure 3, A). In the outpatient group, SF36v2 MCS had a medium correlation with the BDI-II (rho $=-0.64, P<.01)$ and a high correlation with BSRS-5 (rho $=-0.82, P<.01$ ). The BSRS-5 was moderately correlated with the BDI-II (rho $=0.59, P<.01$ ) (Figure 3, B). However, there was no correlation between total sleep quality (PSQI total scores) and all variables, including the severity of PE (Haller index) (Figure 3, B). In the inpatient group, correlations also existed among the SF36v2 MCS, BDI-II, and BSRS-5 as in the outpatient group (Figure 3, C). A medium correlation was found between total sleep quality (PSQI total scores) and psychologic status (rho $=-0.59, P<.01$ for PSQI and MCS; rho $=0.56, P<.01$ for PSQI and BDI-II) (Figure 3, $C$ ).

Table 4 shows the role of all variables in sleep quality (PSQI total scores). The sleep quality of the inpatient group was significantly positively correlated with that of the control group (beta $=2.18, P<.001$ ) in a univariate linear regression analysis. The univariate linear regression analysis also showed that psychologic impairment (BSRS-5 and BDI-II) and daytime functioning impairment (Epworth sleepiness scale and EDS) were significantly positively 


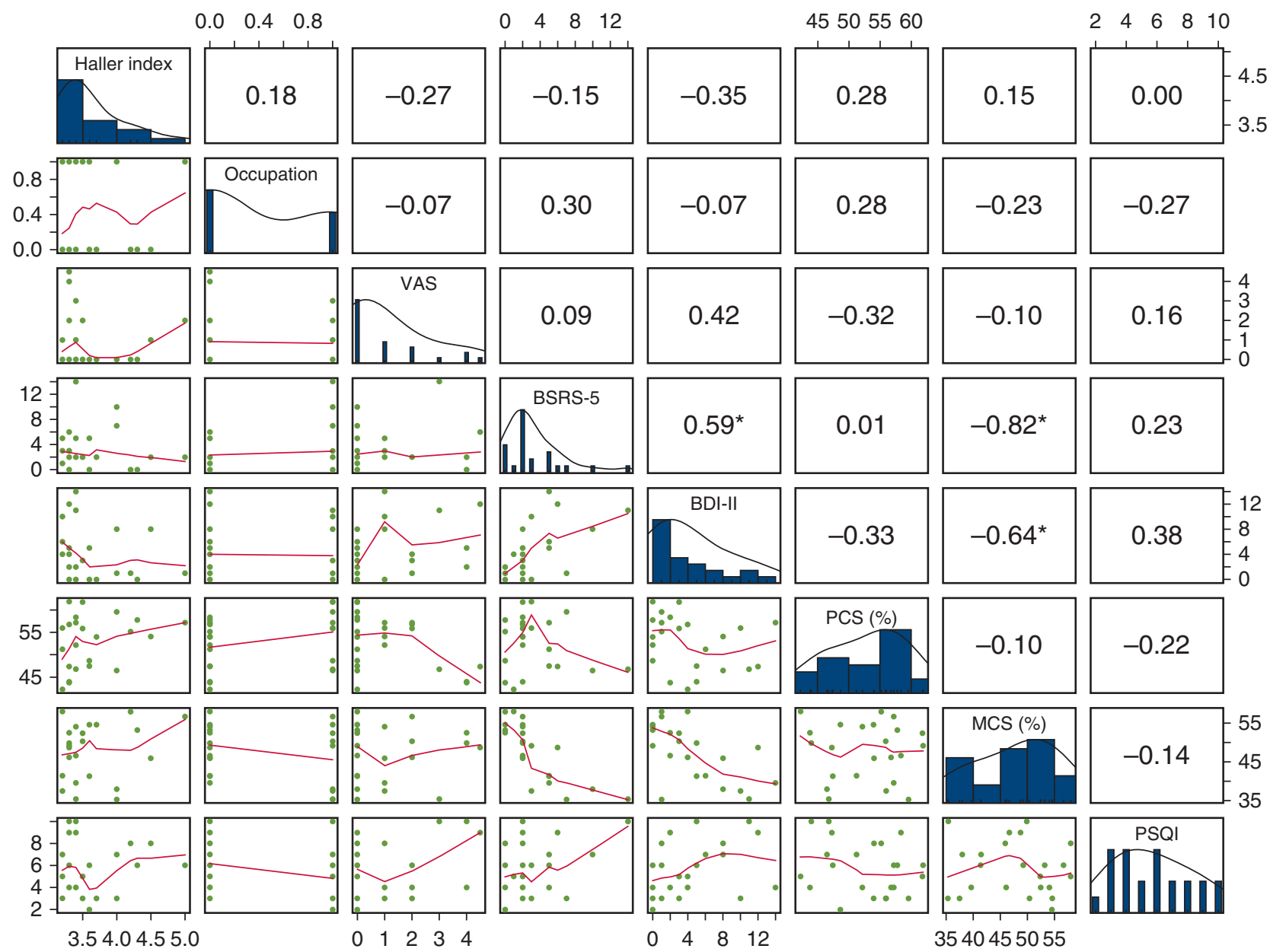

B

FIGURE 3. (continued)

correlated with total sleep quality (all $P<.01$ ). However, all SF-36v2 scales, except PF and BP, showed significant negative correlations with total sleep quality (all $P \leq .01$ ). After using the multivariate linear regression model to simultaneously adjust for those significant factors in a univariate analysis with stepwise selection, the results showed that only total sleep quality was significantly positively correlated with the BDI-II (beta $=0.18, P<.01$ ). There was no correlation between outcome measurement time and total sleep quality.

\section{DISCUSSION}

This study showed that patients with PE had poorer sleep quality, which was associated with their psychologic symptoms but not physical impairments or the severity of PE itself. Pectus deformity has no physiologic relationship with poorer sleep quality. Likewise, sleep quality improved after pectus repair, which was likely related to the psychologic factors. Collectively, this suggests that PE-related psychologic issues affected sleep quality, especially in patients with PE who were waiting for surgical repair.

Research shows that the psychologic impact of PE in children is stronger than its physical impact. ${ }^{1,3,17}$ Zhao and colleagues ${ }^{2}$ found that psychologic trauma in patients with PE was positively correlated with PE severity and age, especially among teenagers in Chinese populations. However, in our study, psychologic trauma was not correlated with disease severity, suggesting PE-related psychologic trauma may differ between children and adults. Adult patients with PE may have come to terms with their chest deformity per se, regardless of its severity.

The surgical indication of our patients was mainly noncosmetic, which was different from previous reports in children. ${ }^{23}$ This indicates the decisive factor for Nuss surgery in adult patients with PE might be different than that for children. 


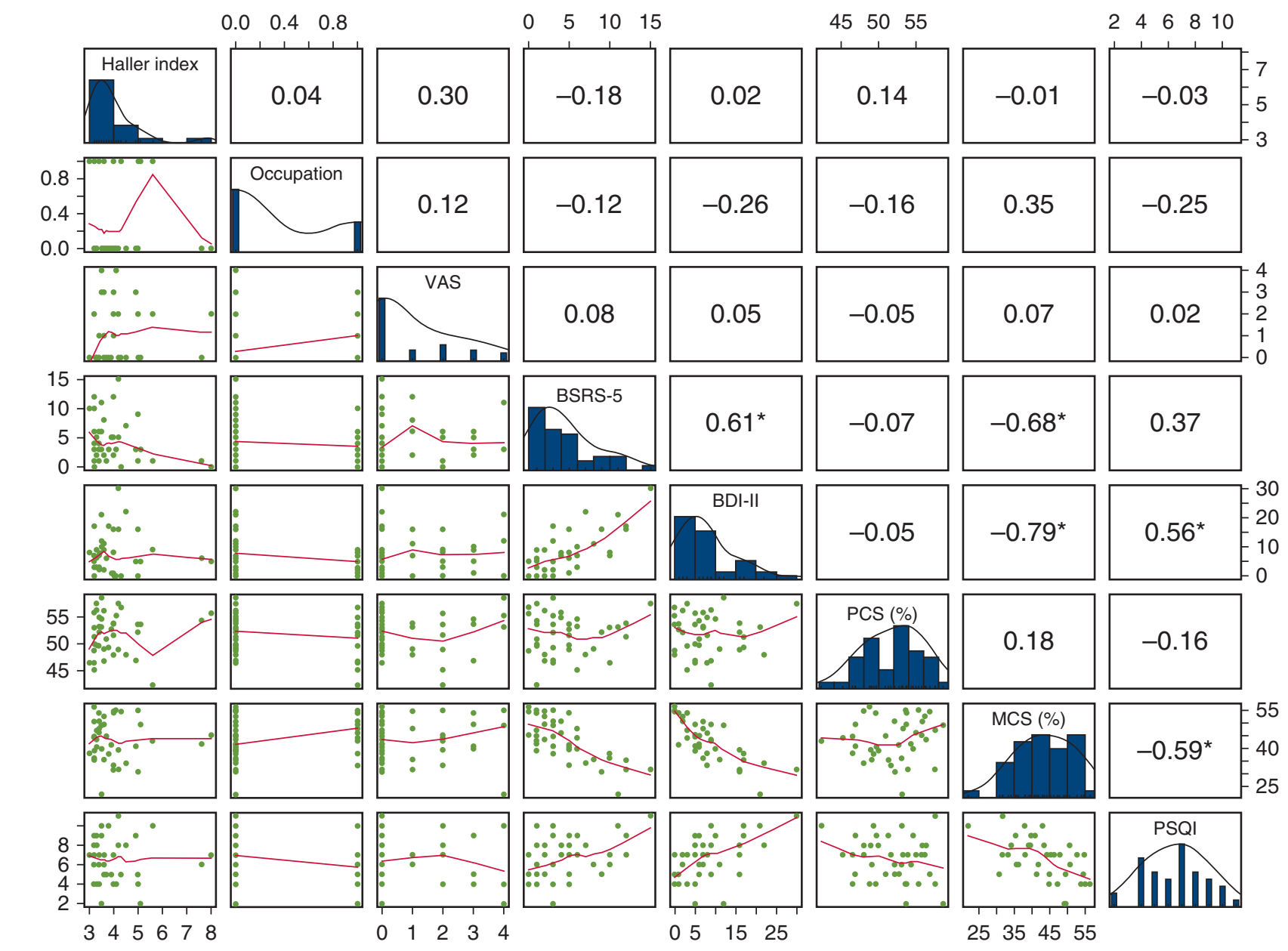

C

FIGURE 3. (continued).

Although the BSRS-5 evaluated psychologic status on the day of measurement, the BDI-II evaluated psychologic status in the week before measurement, and the PSQI evaluated sleep quality in the month before measurement. Because differences were not found between outpatient and inpatient groups on the BSRS-5, but were found on the BDI-II and PSQI, the worse depressive symptoms and poorer sleep quality of our inpatient group might not have been affected by the upcoming surgery. Furthermore, in our multivariate linear regression model, we also showed that outcome measurement time had no role in sleep quality. However, the operation scheduling time in our hospital is usually 1 to 3 months. Thus, the poorer sleep quality of our inpatient group could have been associated with the long wait for surgery, rather than disease severity or psychologic impairment.

The inpatient group showed longer sleep latency than healthy subjects, suggesting they had more difficulty falling asleep after going to bed every night. However, 6 months after surgery, the sleep latency (PSQ-2 scores) did not significantly improve, but the sleep duration (PSQ-3 scores) did significantly improve (became prolonged). This indicated that the inpatient group still had difficulty falling asleep, but they can sleep longer after Nuss surgery. This might be because most patients were asked to convalesce at home and not return to work or school after surgery for 4 to 6 months. In other words, they did not need to work hard or study hard and only took rest (or sleep) at home even during daytime, and therefore they relatively slept "too much," so that their sleep latency was still affected. Further studies might be needed to clarify this issue.

At baseline, only the outpatient group had significantly higher bodily pain sensations than the healthy group. This suggested that adult patients with PE seek surgical correction because of psychologic impairment but not bodily pain. Six months after the operation, even though pain 
TABLE 4. Univariate and multivariate linear regression analysis: Role of variables on PSQI total score

\begin{tabular}{|c|c|c|c|c|}
\hline & \multicolumn{2}{|c|}{ Univariate analysis } & \multicolumn{2}{|c|}{ Multivariate analysis } \\
\hline & Beta $(95 \%$ CI $)$ & $\overline{P \text { value }}$ & Beta $(95 \%$ CI $)$ & $P$ value \\
\hline \multicolumn{5}{|l|}{ Measurement time } \\
\hline Control & 1 & & 1 & \\
\hline Outpatient & $0.98(-0.33,2.29)$ & .140 & $0.31(-0.84,1.46)$ & .594 \\
\hline Inpatient & $2.18(1.01,3.34)$ & $<.001$ & $0.27(-0.95,1.48)$ & .665 \\
\hline \multicolumn{5}{|l|}{ Patients' characteristics } \\
\hline Haller index & $0.05(-0.77,0.88)$ & .896 & & \\
\hline \multicolumn{5}{|l|}{ Gender } \\
\hline Males & 1 & & & \\
\hline Females & $-0.69(-2.13,0.76)$ & .347 & & \\
\hline Age, y & $0.04(-0.06,0.14)$ & .469 & & \\
\hline Body height, $\mathrm{cm}$ & $0.03(-0.04,0.10)$ & .451 & & \\
\hline Body weight, kg & $-0.01(-0.08,0.05)$ & .652 & & \\
\hline Body mass index, $\mathrm{kg} / \mathrm{m}^{2}$ & $-0.10(-0.31,0.12)$ & .380 & & \\
\hline \multicolumn{5}{|l|}{ Occupation } \\
\hline Nonstudent & 1 & & & \\
\hline Student & $-0.62(-1.74,0.50)$ & .272 & & \\
\hline \multicolumn{5}{|l|}{ QOL } \\
\hline VAS scores & $0.31(-0.12,0.75)$ & .159 & & \\
\hline BSRS-5 & $0.37(0.21,0.52)$ & $<.001$ & & \\
\hline BDI-II & $0.24(0.16,0.31)$ & $<.001$ & $0.18(0.09,0.27)$ & $<.001$ \\
\hline \multicolumn{5}{|l|}{ SF-36v2 } \\
\hline PCS, $\%$ & $-0.15(-0.25,-0.05)$ & .003 & & \\
\hline MCS, \% & $-0.15(-0.21,-0.09)$ & $<.001$ & & \\
\hline PF_NBS, $\%$ & $-0.11(-0.23,0.004)$ & .058 & & \\
\hline RP_NBS, \% & $-0.15(-0.21,-0.09)$ & $<.001$ & $-0.09(-0.15,-0.02)$ & .011 \\
\hline BP_NBS, \% & $-0.05(-0.13,0.03)$ & .242 & & \\
\hline GH_NBS, $\%$ & $-0.09(-0.14,-0.04)$ & $<.001$ & & \\
\hline VT_NBS, \% & $-0.15(-0.21,-0.09)$ & $<.001$ & & \\
\hline SF_NBS, $\%$ & $-0.20(-0.29,-0.12)$ & $<.001$ & & \\
\hline RE_NBS, \% & $-0.12(-0.18,-0.06)$ & $<.001$ & & \\
\hline MH_NBS, $\%$ & $-0.14(-0.21,-0.07)$ & $<.001$ & & \\
\hline \multicolumn{5}{|l|}{ Daytime sleepiness } \\
\hline ESS score, points & $0.28(0.16,0.40)$ & $<.001$ & & \\
\hline \multicolumn{5}{|c|}{ Excessive daytime sleepiness } \\
\hline No, ESS score $\leq 10$ & 1 & & & \\
\hline Yes, ESS score $>10$ & $2.43(0.98,3.88)$ & .001 & & \\
\hline
\end{tabular}

Bold indictes significantly different while $P<.01$. CI, Confidence interval; $Q O L$, quality of life; VAS, visual analog scale; $B S R S-5$, Brief Symptom Rating Scale; BDI-II, Beck Depression Inventory; $S F-36 v 2$, 36-Item Short Form Survey Chinese Version 2; PCS, physical component score; $M C S$, mental component score; $P F$, physical functioning; $N B S$, norm-based score (0-100\%); RP, role physical; $B P$, bodily pain; $G H$, general health; $V T$, vitality; $S F$, social functioning; $R E$, role emotional; $M H$, mental health; $E S S$, Epworth sleepiness scale.

was worse in the inpatient group, the sleep quality improved, indicating bodily pain did not affect patients' sleep quality.

Previous studies revealed both impaired physical and psychologic function of children and adult patients with PE can be improved by Nuss surgery. ${ }^{4,5,18,19}$ We also observed the improvement of psychologic and physical function in our adult patients with PE.

The PSQI used in this study only evaluated subjective sleep quality. Polysomnography (PSG) is a well-known objective measure of sleep quality that yields more detailed information about sleep. We suggest PSG might be used to evaluate objective sleep quality before and after
Nuss surgery. In addition, PSG can evaluate sleepdisordered breathing, especially obstructive sleep apnea (OSA). As is well known, laryngomalacia is found to be associated or present with OSA ${ }^{24,25}$ and PE. ${ }^{26,27}$ Similar to laryngomalacia, patients with OSA experience upper airway obstruction resulting in chest retraction during inspiration. However, only limited studies have evaluated the association between OSA and PE. In 1992, Castiglione and colleagues ${ }^{28}$ reported that $82 \%$ of patients with OSA children presented with PE. In 2016, Ma and colleagues $^{29}$ reported a 5-year-old child with previously known persistent snoring during sleep for more than 4 years experienced severe sternum depression mimicking 
PE during an episode of upper airway infection. The PSG revealed he had severe OSA. His severe OSA and sternal depression dramatically improved after a standard adenotonsillectomy, which was done only to correct his OSA but not his PE. ${ }^{29}$ They suggested OSA might aggravate sternal depression, and sternal depression might persist in mimicking PE if OSA is not treated over a long period. ${ }^{29}$ This suggests that OSA might be a risk factor or aggravating factor for PE that may necessitate a second Nuss procedure. Therefore, it might be helpful to perform a PSG for evaluated sleep-disordered breathing before and after Nuss surgery. Patients with PE who also have or present with OSA might receive more frequent follow-ups after removal of the Nuss bar to detect the possibility of recurring $P E$.

Most previous studies have used a modified Nuss questionnaire or other disease-specific questionnaires to evaluate the effect of Nuss surgery; only 1 study used the SF-36 to evaluate the QOL of patients with PE aged 14 to 35 years. ${ }^{11}$ They found that regardless of age, patients with PE attained significantly lower PCS scores than the general population (Cohen's $d=0.5$ ), but their MCS scores did not differ from general population. ${ }^{11}$ In contrast, our results show that PCS and MCS scores in patients with PE were significantly lower than those of healthy adults. Perhaps this could be understood as the higher degree of PE-related psychologic trauma experienced by adult patients compared with children.

Surgical indications to correct PE are generally based on clinical judgments and physical problems. ${ }^{30}$ They tend to be treated as cosmetic solutions for children and noncosmetic for adults, ${ }^{23,31-33}$ which may explain why surgical indications of our patients were mainly noncosmetic: They experienced more pain and worse QOL and sleep quality than the general population.

Further, because patients' psychologic problems outweigh physical problems, some investigators suggest that psychologic problems should be considered as surgical indicators. ${ }^{2,3,17}$ In our study, we showed that poor sleep quality was associated with PE-related psychologic problems in patients with PE who had already decided to receive Nuss surgery. Therefore, we suggest that routine evaluation of sleep quality could be considered before and after surgical correction for PE.

\section{Study Limitations}

This study had many limitations. First, our study sample was small and therefore may not be representative of all patients with PE. Second, convenience sampling was used for recruiting the control group. Because it primarily comprised medical staff, the control group might be not representative of the general population. Third, all questionnaires were self-reports. Although participants received assistance to ensure questionnaire integrity, psychologic counselors or psychiatrists were not present to facilitate or confirm the trueness of all responses. Fourth, the measurement time and environment of these 3 groups were different. The control group was measured at our office with only research assistant to ensure the questionnaire integrity. The outpatient group was measured at the outpatient department, where time is important; therefore, they did not have much time to complete the measurements. The inpatient group was measured in the ward, where they had enough time to complete all the measurements with a trained research assistant. Further, all SF-36v2 scores were presented as norm-based scores using the general US population of 2009 for comparison. This may not be the best group for comparison because our study subjects were all Taiwanese: There may be racial and cultural differences in physical and psychosocial function. Moreover, we did not have a comparison group with a chronic health condition or another group scheduling another surgery as comparisons. The sleep disturbances might not be a physiologic problem specific to PE, but a more global psychologic problem associated with chronic disease or any other surgery. Finally, only the inpatient group had a postoperative (ie, 6-month) measurement; there was no 6-month measurement in the control group or outpatient group. The measurements of all 3 groups might indicate temporary changes among individuals. Further, well-designed studies are needed on this issue.

\section{CONCLUSIONS}

Our findings suggest a potential association between poor sleep quality and PE-related psychologic impairment, but not the disease per se. Patients' sleep quality should be evaluated before and after the Nuss procedure.

\section{Conflict of Interest Statement}

Authors have nothing to disclose with regard to commercial support.

\section{References}

1. Ji Y, Liu W, Chen S, Xu B, Tan Y, Wang X, et al. Assessment of psychosocial functioning and its risk factors in children with pectus excavatum. Health Qual Life Outcomes. 2011;9:28.

2. Zhao J, Luo L, Xiao LJ, Gu LY, Sun TS. Psychological trauma of funnel chest in adolescents and the appropriate age for minimally invasive surgery repair. Chin Med J (Engl). 2013;126:2876-80.

3. Koumbourlis AC. Pectus excavatum: pathophysiology and clinical characteristics. Paediatr Respir Rev. 2009;10:3-6.

4. Kuru P, Bostanci K, Ermerak NO, Bahadir AT, Afacan C, Yuksel M. Quality of life improves after minimally invasive repair of pectus excavatum. Asian Cardiovasc Thorac Ann. 2015;23:302-7.

5. Krasopoulos G, Dusmet M, Ladas G, Goldstraw P. Nuss procedure improves the quality of life in young male adults with pectus excavatum deformity. Eur J Cardiothorac Surg. 2006;29:1-5.

6. Hanna WC, Ko MA, Blitz M, Shargall Y, Compeau CG. Thoracoscopic Nuss procedure for young adults with pectus excavatum: excellent midterm results and patient satisfaction. Ann Thorac Surg. 2013;96:1033-8.

7. Ferrell BR, Dow KH, Grant M. Measurement of the quality of life in cancer survivors. Qual Life Res. 1995;4:523-31.

8. Ferrell BR, Grant M, Funk B, Garcia N, Otis-Green S, Schaffner ML. Quality of life in breast cancer. Cancer Pract. 1996;4:331-40. 
9. Roberts J, Hayashi A, Anderson JO, Martin JM, Maxwell LL. Quality of life of patients who have undergone the Nuss procedure for pectus excavatum: preliminary findings. J Pediatr Surg. 2003;38:779-83.

10. Kim HK, Shim JH, Choi KS, Choi YH. The quality of life after bar removal in patients after the Nuss procedure for pectus excavatum. World J Surg. 2011; 35:1656-61.

11. Steinmann C, Krille S, Mueller A, Weber P, Reingruber B, Martin A. Pectus excavatum and pectus carinatum patients suffer from lower quality of life and impaired body image: a control group comparison of psychological characteristics prior to surgical correction. Eur J Cardiothorac Surg. 2011;40:1138-45.

12. Buysse DJ, Reynolds CF III, Monk TH, Berman SR, Kupfer DJ. The Pittsburgh sleep quality index: a new instrument for psychiatric practice and research. Psychiatry Res. 1989;28:193-213.

13. Iliescu EA, Coo H, McMurray MH, Meers CL, Quinn MM, Singer MA, et al. Quality of sleep and health-related quality of life in haemodialysis patients. Nephrol Dial Transplant. 2003;18:126-32.

14. Shen Q, Huang X, Luo Z, Xu X, Zhao X, He Q. Sleep quality, daytime sleepiness and health-related quality-of-life in maintenance haemodialysis patients. J Int Med Res. 2016;44:698-709.

15. Beck AT, Steer RA, Ball R, Ranieri W. Comparison of Beck depression inventories -IA and -II in psychiatric outpatients. J Pers Assess. 1996;67:588-97.

16. Lee MB, Liao SC, Lee YJ, Wu CH, Tseng MC, Gau SF, et al. Development and verification of validity and reliability of a short screening instrument to identify psychiatric morbidity. J Formos Med Assoc. 2003;102:687-94.

17. Koumbourlis AC. Pectus deformities and their impact on pulmonary physiology. Paediatr Respir Rev. 2015;16:18-24.

18. Lawson ML, Cash TF, Akers R, Vasser E, Burke B, Tabangin M, et al. A pilot study of the impact of surgical repair on disease-specific quality of life among patients with pectus excavatum. J Pediatr Surg. 2003;38:916-8.

19. Kelly RE Jr, Cash TF, Shamberger RC, Mitchell KK, Mellins RB, Lawson ML, et al. Surgical repair of pectus excavatum markedly improves body image and perceived ability for physical activity: multicenter study. Pediatrics. 2008;122: 1218-22.

20. Bimler HP. Bimler therapy. Part 1. Bimler cephalometric analysis. J Clin Orthod. 1985;19:501-23.
21. Chiba N. Effects of in vitro antibiotic resistance on treatment: bismuthcontaining regimens. Can J Gastroenterol. 2000;14:885-9.

22. Chan SF, Chen TH, Liao YM, Chou KR, Tsai PS. Development and preliminary validation of the Chinese version of the sleep-associated monitoring index. Int $J$ Nurs Stud. 2012;49:54-64.

23. Kabbaj R, Burnier M, Kohler R, Loucheur N, Dubois R, Jouve JL. Minimally invasive repair of pectus excavatum using the Nuss technique in children and adolescents: indications, outcomes, and limitations. Orthop Traumatol Surg Res. 2014;100:625-30

24. Digoy GP, Shukry M, Stoner JA. Sleep apnea in children with laryngomalacia: diagnosis via sedated endoscopy and objective outcomes after supraglottoplasty. Otolaryngol Head Neck Surg. 2012;147:544-50.

25. Oomen KP, Modi VK. Occult laryngomalacia resulting in obstructive sleep apnea in an infant. Int J Pediatr Otorhinolaryngol. 2013;77:1617-9.

26. Schaerer D, Virbalas J, Willis E, Siegel B, Gonik N, Bent J. Pectus excavatum in children with laryngomalacia. Int J Pediatr Otorhinolaryngol. 2013;77:1721-3.

27. Avelino MA, Liriano RY, Fujita R, Pignatari S, Weckx LL. Treatment laryngomalacia: experience with 22 cases. Braz J Otorhinolaryngol. 2005;71:330-4.

28. Castiglione N, Eterno C, Sciuto C, Bottaro G, La Rosa M, Patane R. The diagnostic approach to and clinical study of 23 children with an obstructive sleep apnea syndrome. Pediatr Med Chir. 1992;14:501-6.

29. Ma W, Wang J, Xie Y. Children severe OSAHS with pectus excavatum: a case report. Lin Chung Er Bi Yan Hou Tou Jing Wai Ke Za Zhi. 2016;30:407-9.

30. Nuss D, Kelly RE Jr, Croitoru DP, Katz ME. A 10-year review of a minimally invasive technique for the correction of pectus excavatum. J Pediatr Surg. 1998;33:545-52.

31. Bay V, Farthmann E, Naegele U. Unoperated funnel chest in middle and advances age: evaluation of indications for operation. J Pediatr Surg. 1970;5:606-9.

32. Mansour KA, Thourani VH, Odessey EA, Durham MM, Miller JI Jr, Miller DL. Thirty-year experience with repair of pectus deformities in adults. Ann Thorac Surg. 2003;76:391-5.

33. Fonkalsrud EW, DeUgarte D, Choi E. Repair of pectus excavatum and carinatum deformities in 116 adults. Ann Surg. 2002;236:304-14.

Key Words: pectus excavatum, sleep quality 
TABLE E1. List of acronyms

\begin{tabular}{|c|c|c|c|}
\hline Acronyms & Expansions & Meanings & Scores \\
\hline BSRS-5 & Brief Symptom Rating Scale & Psychologic status on the day & $0-20$ \\
\hline BDI-II & Beck Depression Inventory version 2 & Psychologic status in recent $1 \mathrm{wk}$ & $0-63$ \\
\hline SF-36 & 36-Item Short-Form Survey & Health-related quality of life evaluation in recent $1 \mathrm{mo}$ & \\
\hline PCS & Physical component score of SF-36 & $\begin{array}{l}\text { Limitation in self care, physical, social, and role activities; severe } \\
\text { bodily pain, frequent tiredness; health rated poor or excellent }\end{array}$ & $0 \%-100 \%$ \\
\hline MCS & Mental component score of SF-36 & $\begin{array}{l}\text { Frequent psychosocial distress, substantial social and role disability } \\
\text { due to emotional problems; health in general rated poor or } \\
\text { excellent }\end{array}$ & $0 \%-100 \%$ \\
\hline $\mathrm{PF}$ & Physical functioning & $\begin{array}{l}\text { Performance of physical activities (self-care, walking, and vigorous } \\
\text { physical activities) }\end{array}$ & $0 \%-100 \%$ \\
\hline $\mathrm{RP}$ & Role physical & $\begin{array}{l}\text { How a person's typical role activities (eg, childcare, job) are limited } \\
\text { by physical health }\end{array}$ & $0 \%-100 \%$ \\
\hline $\mathrm{BP}$ & Bodily pain & $\begin{array}{l}\text { Intensity, duration, and frequency of bodily pain (limitations in usual } \\
\text { activities due to pain) }\end{array}$ & $0 \%-100 \%$ \\
\hline $\mathrm{GH}$ & General health & The beliefs and evaluations of a person's overall health & $0 \%-100 \%$ \\
\hline VT & Vitality & Feelings of energy, the absence of fatigue & $0 \%-100 \%$ \\
\hline $\mathrm{SF}$ & Social functioning & $\begin{array}{l}\text { How a person develops and maintains social relationships (eg, with } \\
\text { family, friends) }\end{array}$ & $0 \%-100 \%$ \\
\hline $\mathrm{RE}$ & Role emotional & $\begin{array}{l}\text { How a person's typical role activities (eg, childcare, job) limited by } \\
\text { emotional problems }\end{array}$ & $0 \%-100 \%$ \\
\hline MH & Mental health & A person's emotional, cognitive, and intellectual status & $0 \%-100 \%$ \\
\hline PSQI & Pittsburgh Sleep Quality Index & Sum of PSQ-1 to PSQ-7; total sleep quality in recent $1 \mathrm{mo}$ & $0-21$ \\
\hline PSQ-1 & PSQI question 1 & Subjective sleep quality & $0-3$ \\
\hline PSQ-2 & PSQI question 2 & Subjective sleep latency & $0-3$ \\
\hline PSQ-3 & PSQI question 3 & Subjective sleep duration & $0-3$ \\
\hline PSQ-4 & PSQI question 4 & Subjective sleep efficiency & $0-3$ \\
\hline PSQ-5 & PSQI question 5 & Subjective sleep disturbance & $0-3$ \\
\hline PSQ-6 & PSQI question 6 & Sleep pill use frequency & $0-3$ \\
\hline PSQ-7 & PSQI question 7 & Daytime functional impairment & $0-3$ \\
\hline ESS & Epworth Sleepiness Scale & Excessive daytime sleepiness, subjective daytime functioning & $0-24$ \\
\hline
\end{tabular}

